\title{
Tradurre la scienza in strategie politiche per migliorare in Europa la cura dei pazienti con ADPKD
}

\author{
Luisa Sternfeld Pavia \\ Presidente Associazione Italiana del Rene Policistico AIRP onlus, Milano
}

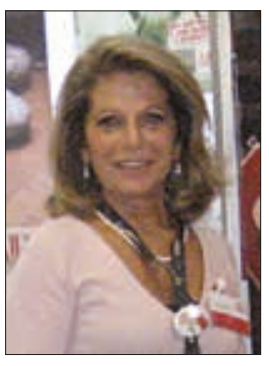

Luisa Sternfeld Pavia
Bruxelles, 29 gennaio 2015

La giornata ha segnato un momento importante: la pubblicazione di una relazione a cura dell'European ADPKD Forum (EAF), gruppo multidisciplinare internazionale formato da esperti nei campi della nefrologia, epatologia e genetica e da rappresentanti di associazioni europee per la tutela dei malati, impegnati a migliorare la salute e la qualità della vita delle persone affette da malattia policistica renale autosomica dominante (Autosomal Dominant Polycystic Kidney Disease, ADPKD), patologia ereditaria progressiva cronica caratterizzata dalla crescita di cisti contenenti liquido nei reni e nel fegato (1).

La pubblicazione, intitolata "Tradurre le conoscenze in strategie politiche per migliorare la cura della malattia policistica renale autosomica dominante in Europa", (Fig. 1) descrive dettagliatamente l'importanza del ruolo degli operatori sanitari quali attori volti a promuovere lo sviluppo e l'erogazione di cure migliori per l'ADPKD e offre uno spaccato esaustivo della realtà europea in termini di gestione della malattia.

La pubblicazione evidenzia la disparità a livello di standard clinici in Europa (2) e rileva la necessità di promuovere la formazione e il supporto dei medici non specialisti, in modo da metterli in condizione di migliorare

Accepted: February 26, 2015

Published online: April 7, 2015

Indirizzo per la corrispondenza:

Dr.ssa Luisa Sternfeld Pavia

AIRP Associazione Italiana Rene Policistico onlus

Via A. Bazzini 2

20131 Milano

luisa.sternfeld.airp@renepolicistico.it l'accuratezza e l'adeguatezza della diagnosi e del trattamento dell'ADPKD e dei sintomi ad essa associati.

Tali sintomi comprendono dolore, infezioni delle cisti e del tratto urinario, calcoli renali, ematuria e distensione addominale (3-8). I pazienti colpiti da ADPKD possono altresì presentare sintomi cardiovascolari, in quanto a rischio elevato di sviluppare ipertensione e malattie cardiovascolari (1).

Il volume evidenzia inoltre che i reni policistici conducono a un quadro di insufficienza renale nella maggior

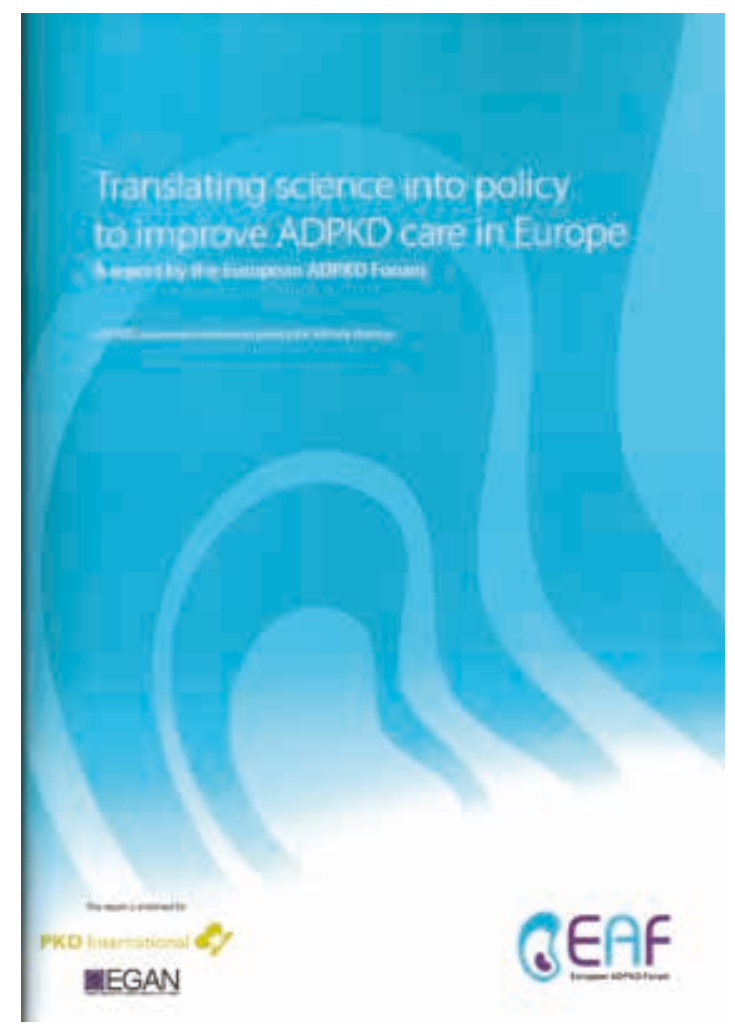

Fig. 1 - Documento di Bruxelles. 


\section{European ADPKD Forum members}

\begin{tabular}{|c|c|}
\hline \multicolumn{2}{|l|}{ Co-Chairs } \\
\hline Dr Fichond Somathond & Unversty at Cimbridge/Mddenbrockes Horpita, Cambridge, UX. \\
\hline Tess Hawil & PD retervitionst Landon, UK \\
\hline \multicolumn{2}{|c|}{ All participants by wecialty } \\
\hline \multicolumn{2}{|c|}{ Nepherotosy } \\
\hline Phet, Cevier Devupst & Unwersy of 7 furch fursh Swltheriang \\
\hline Phor. Insk Eeder & 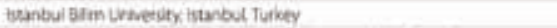 \\
\hline Dr Aan 7 Canseveort & Unversty Mefical Center Gronivgen Goringer: Wethelands \\
\hline Dr loset tuis Gemb & 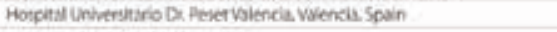 \\
\hline Prol Abert on & Unwenty at Sheffeld, Sheffed, ur \\
\hline And Mesispron & 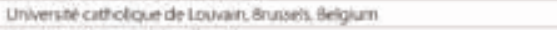 \\
\hline Prod, Wicente Doures & Maro Clak, foctenter, Nhe Ush \\
\hline Mor Gend Wate & 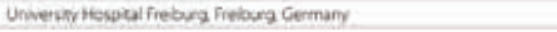 \\
\hline \multicolumn{2}{|l|}{ Hepatalogy } \\
\hline Phed, foost Dventh & 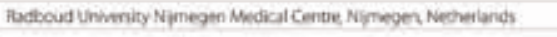 \\
\hline \multicolumn{2}{|l|}{ Gemertics } \\
\hline Dr Aichord Sonatond & 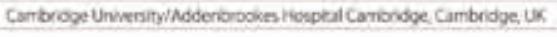 \\
\hline \multicolumn{2}{|l|}{ Metiant advotacy } \\
\hline Bvendo de Coninck & 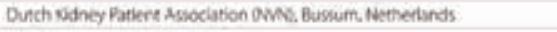 \\
\hline Pess Marris & PRO rservitiond London UK \\
\hline Alostair Kent & 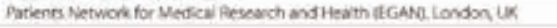 \\
\hline
\end{tabular}

The authons thark DI Kaven Facey, Unwersty of Gasgow, Gasgon, UK, for her review and conkribution to Sertion 7 .

\section{Polycystic kidney disease organisations}

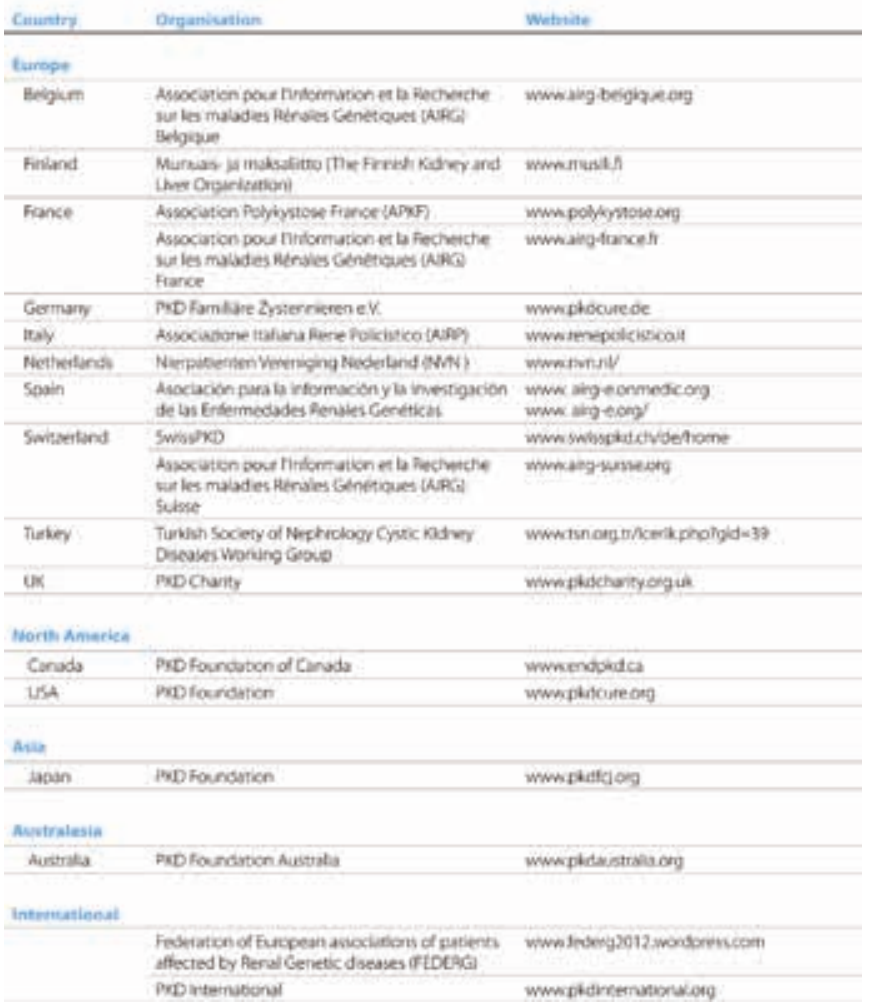

Fig. 2 - Membri di EAF e le Associazioni di Pazienti che ne fanno parte. parte dei pazienti $(2,9)$ e che rallentare il decorso della malattia comporterebbe esiti migliori per il paziente e risultati positivi in termini di efficienza per la sanità.

Salutando la pubblicazione, il Dr. Richard Sandford, Consultant Clinical Geneticist presso l'Addenbrooke's Hospital di Cambridge e co-presidente dell'EAF, così si è espresso: "Attualmente la cura dell'ADPKD è incentrata sulla gestione generale del degrado della funzione renale e dei sintomi legati alla malattia. Al momento non esistono terapie specifiche per la malattia. Nell'UE, i percorsi terapeutici sono frammentari e si registra una limitata conoscenza della malattia a livello specialistico. Occorre individuare soluzioni innovative a lungo termine, al fine di migliorare le condizioni dei pazienti e rispondere alle sfide finanziarie e sociali poste dall'ADPKD».

La relazione dell'EAF viene pubblicata con il patrocinio di due importanti associazioni per la tutela dei malati: PKD International e la Patients Network for Medical Research and Health (EGAN).

Le seguenti raccomandazioni, riprese nella "Dichiarazione di Bruxelles su ADPKD", documento a sé stante, che vede la luce oggi contestualmente alla relazione e rappresenta il "piano d'azione strategico dell'Unità Europea" nella lotta contro la malattia, mirano ad affrontare le attuali necessità di tutti i pazienti affetti da ADPKD in Europa:

- Implementazione di approcci terapeutici graduali I Governi sono chiamati a sostenere lo sviluppo di un approccio graduale, coordinato a livello nazionale, alla cura dell'ADPKD avvalendosi della collaborazione di esperti, associazioni per la tutela dei malati e altri soggetti di riferimento, in sintonia con la priorità politica, della Commissione Europea (CE), di affrontare le disparità in ambito sanitario.

- Istituzione di centri specialistici per la cura dell'ADPKD

Si auspica la creazione di una rete europea di centri specialistici per la cura dell'ADPKD, al fine di facilitare la ricerca e favorire percorsi di cura armonizzati, integrati e incentrati sul paziente, a garanzia del miglior sviluppo e uniformità delle cure.

- Sostegno alle innovazioni terapeutiche

La CE e i Governi nazionali sono chiamati a sostenere la ricerca al fine di sviluppare trattamenti atti a modificare il decorso della malattia e potenzialmente in grado di mantenere la qualità della vita, ritardare il declino della funzione renale e migliorare l'aspettativa di vita dei pazienti, riducendo altresì l'impatto economico sui sistemi sanitari.

\section{- Privilegiare la prognosi}

I Governi e il mondo della sanità sono chiamati a implementare di routine metodi atti a valutare la prognosi nei pazienti con ADPKD, al fine di informare 
l'iter decisionale clinico, la ricerca e l'innovazione.

\section{- Empowerment dei pazienti}

I vari soggetti di riferimento - Commissione Europea, Governi nazionali e Sanità - sono chiamati a fornire un'informazione migliore ai singoli pazienti affetti da ADPKD e ai loro familiari, coinvolgendo altresì le associazioni per la tutela dei malati nelle decisioni riguardanti la pianificazione e l'assistenza sanitaria per l'ADPKD.

- Coinvolgimento dei pazienti nell'iter di valutazione delle tecnologie sanitarie

Le organizzazioni incaricate della valutazione delle tecnologie sanitarie (Health Technology Assessment, HTA) sono chiamate a impegnarsi al fine di coinvolgere le associazioni per la tutela dei malati di ADPKD nei rispettivi processi di valutazione, al fine di poter contare sulle loro conoscenze specifiche in merito alle difficoltà di chi convive con l'ADPKD, e sulle loro aspirazioni relativamente a nuovi trattamenti, secondo gli standard qualitativi di HTA International in materia di partecipazione dei pazienti ai processi di valutazione delle tecnologie sanitarie.

ADPKD ha conseguenze rilevanti anche sulla qualità della vita dei pazienti che manifestano comunemente stati di dolore da acuto a cronico (3-8) e sono maggiormente predisposti a condizioni di ansia e depressione rispetto alla popolazione generale (10-12). Inoltre, come si riporta dettagliatamente nella relazione, i risultati emersi da una ampia indagine, condotta fra 730 pazienti di ADPKD in Europa, evidenziano l'impatto negativo che la malattia ha sui familiari ( $77 \%$ degli intervistati), sui rapporti interpersonali (41\%), a livello di rapporti sessuali $(42 \%)$, sulle attività sociali ( $33 \%)$ e sulle decisioni riguardanti la procreazione (35\%) (Fig. 2).

Entro breve sarà disponibile la traduzione del volume

"Translating science into policy to improve ADPKD care in Europe - A report by the European ADPKD Forum (EAF)".

\section{Disclosures}

Financial support: No financial support was received for this submission.

Conflict of interest: The author has no conflict of interest.

\section{Bibliografia}

1. Torres VE, Harris PC, Pirson Y. Autosomal dominant polycystic kidney disease. Lancet 2007;369:1287-1301.

2. Spithoven EM, Kramer A, Meijer E, et al. Analysis of data from the ERA-EDTA Registry indicates that conventional treatments for chronic kidney disease do not reduce the need for renal replacement therapy in autosomal dominant polycystic kidney disease. Kidney Int 2014;86(6):1244-1252.

3. Bajwa ZH, Sial KA, Malik AB, Steinman TI. Pain patterns in patients with polycystic kidney disease. Kidney Int 2004;66: 1561-1569.

4. Oberdhan D, et al. Patient-reported pain in autosomal dominant polycystic kidney disease: initial concepts based on patient focus group discussions [Abs SA-PO283]. J Am Soc Nephrol 2013;24 Abs Suppl:692.

5. Oberdahn D, et al. Patient experience with pain related to autosomal dominant polycystic kidney disease (ADPKD) [Abs PUB285]. J Am Soc Nephrol 2014;25 Abs Suppl:960.

6. Miskulin DC, Abebe KZ, Chapman AB, et al. Health-related quality of life in patients with autosomal dominant polycystic kidney disease and CKD Stages 1-4: a cross-sectional study. Am J Kidney Dis 2014;63:214-226.

7. Heiwe S, Bjuke M. "An evil heritage": interview study of pain and autosomal dominant polycystic kidney disease. Pain Manag Nurs 2009;10:134-141.

8. Casteleijn NF, Visser FW, Drenth JP, et al. A stepwise approach for effective management of chronic pain in autosomaldominant polycystic kidney disease. Nephrol Dial Transplant 2014;29 (Suppl 4):iv142-153.

9. Shaw C, Simms RJ, Pitcher D, Sandford R. Epidemiology of patients in England and Wales with autosomal dominant polycystic kidney disease and end-stage renal failure. Nephrol Dial Transplant 2014; 29:1910-1918.

10. Spithoven EM, et al. Renal replacement therapy for autosomal dominant polycystic kidney disease (ADPKD) in Europe: prevalence and survival-an analysis of data from the ERAEDTA Registry. Nephrol Dial Transplant 2014;29 (Suppl 4): iv15-iv25.

11. Wijnands TF, Neijenhuis MK, Kievit W, et al. Evaluating healthrelated quality of life in patients with polycystic liver disease and determining the impactof symptoms and liver volume. Liver Int 2014;34:1578-1583.

12. de Barros BP, Nishiura JL, Heilberg IP, Kirsztajn GM. Anxiety, depression, and quality of life in patients with familial glomerulonephritis or autosomal dominantpolycystic kidney disease. J Bras Nefrol 2011;33:120-128. 